Pacific Journal of Mathematic 


\title{
IN SEARCH OF NONSOLVABLE GROUPS OF CENTRAL TYPE
}

\author{
Robert A. Liebler ANd Jay E. Yellen
}

\begin{abstract}
In 1963 Iwahori and Matsumoto conjectured that a finite group possessing a central simple projective group algebra must be solvable. We verify this conjecture in case all composition factors are known simple groups.
\end{abstract}

1. Introduction. A natural question in the theory of projective group representations is which finite groups $\bar{G}$ possess a projective group algebra $A$ that has the simplest possible structure. Iwahori and Matsumoto [10] conjectured that $\bar{G}$ must be solvable if $A$ is central simple. DeMeyer and Janusz [2, Theorem 1] showed that such a group possesses a central extension $G$ (of central type) such that there is a complex irreducible character $\chi$ of $G$ such that $\chi(1)^{2}=[G: Z(G)]$. DeMeyer and Janusz also provided the first support for the solvability conjecture.

In this paper we continue the work of these authors and Isaacs [6], Gagola [4] and Yellen [14] and show

MAIN THEOREM. A nonsolvable group of central type must possess a new simple group as a composition factor.

We consider the following hypotheses on an arbitrary finite group $S$ :

(1.1) Hypothesis. There is a prime $p$ such that $S$ has a nontrivial abelian Sylow $p$-subgroup and $p \nmid \mid$ Out $S \mid$.

(1.2) Hypothesis. If there is a proper subgroup $I$ of $p$-power index, then $I$ is nonsolvable and all composition factors of $I$ satisfy hypothesis (1.1).

Hypothesis (1.1) is satisfied by all known simple groups (3.1) and (1.2) is also satisfied by all known nonabelian simple groups except for (certain) PSL $(2, q)$ (3.2). Theorem 2.6 shows a group of central type having minimal order among those that are nonsolvable and have no composition factor failing (1.1) must have a composition factor $S$ that fails (1.2). Theorem 2.7 shows further that $S$ cannot be a PSL $(2, q)$ and the main theorem follows.

Our notation is standard and follows Gagen [3], Huppert [5] and Isaacs [7] when appropriate. 
The authors thank F. R. DeMeyer, J. I. Hall, W. M. Kantor and E. E. Shult for several helpful discussions. We also thank the referee for suggesting "abelian" rather than "cyclic" in (1.1).

2. The structure of a group of central type.

THEOREM 2.1. Let $G$ be a group of central type. Then no component of $G$ satisfies hypothesis (1.1).

Proof. Suppose $G$ has a component $E_{1}$ satisfying (1.1) and let $Z=Z(G)$. Label the $G$-conjugates of $E_{1}$ as $E_{1} \cdots E_{m}$ and set $K=$ $\left\langle E_{1} \cdots E_{m}\right\rangle Z$.

Let $R$ be a Sylow $p$-subgroup of $G$ containing $P$, a Sylow $p$ subgroup of $K$ and set $N=\cap N_{R}\left(E_{i}\right)$. Then $[R: N] \mid m !$, since $R$ acts as a permutation group on $\left\{E_{1} \cdots E_{m}\right\}$ and $N$ is the kernel of this action.

Take $x \in N$. By hypothesis (1.1), $\left\langle x, P \cap E_{i}\right\rangle$ induces the same group of automorphisms of $S=E_{i} / Z\left(E_{i}\right)$ as does $P \cap E_{i}$. Hence there is $x_{i} \in E_{i} \cap P$ such that $x x_{i} \in C_{G}\left(E_{i} / Z\left(E_{i}\right)\right)$. However $C_{G}\left(E_{i} / Z\left(E_{i}\right)\right)=$ $C_{G}\left(E_{i}\right)$ by $[3,10.3 \mathrm{a}]$, and $\left[x x_{i}, P \cap E_{i}\right]=1$, so $x x_{i} \in C_{R}\left(P \cap E_{i}\right)$. Similarly $\left[x_{i}, x_{j}\right]=1$ for $i \neq j$, by $[3,10.2 \mathrm{a}]$. It follows that $x x_{1} \cdots x_{m} \in C_{R}(P)$. This shows $N=C_{R}(P)$, since $P$ itself is abelian $[3,10.2 \mathrm{a}]$.

Now a theorem of DeMeyer and Janusz [2, Theorem 2] implies that $R$ is a group of central type with center $Z \cap R$. Take $\chi \in$ char $R$ and $\zeta \in$ char $Z \cap R$ to be the associated characters, so $\left.\chi\right|_{\left.\right|_{n R}}=$ $[R: Z \cap R]^{1 / 2} \zeta$. Let $\tau$ be an irreducible constitutent of the induced character $\zeta^{P}$. By Clifford's theorem $[8,17.3][R: \mathscr{J}(\tau)]=[P: Z \cap R]$, as $P^{\prime}=1$. But $\mathscr{J}_{R}(\tau) \geqq C_{R}(P)=N$, so we have

$$
p^{m}|[P: Z \cap R]|[R: N] \mid m !
$$

which is absurd. This proves (2.1).

In order to minimize repitition we fix the following notation for the rest of this section. Let $G$ be a nonsolvable group of central type having minimal order among those possessing only composition factors satisfying (1.1). The characters $\chi \in \operatorname{char} G$ and $\zeta \in \operatorname{char} Z$, $Z=Z(G)$ are supposed to satisfy $\left.\chi\right|_{Z}=[G: Z]^{1 / 2 \zeta}$. Take $K$ to be a minimal normal subgroup of $G$ among those properly containing $Z$ and take $\tau \in \operatorname{char} K$ to be an irreducible constituent of the induced character $\zeta^{K}$. 
Proof. By Theorem $2.1 K / Z$ is an elementary abelian $p$-group for some prime $p$. Consider the "bilinear" function $\langle\rangle:, K \times K \rightarrow C^{*}$ defined by $\langle\langle x, y\rangle=\zeta([x, y])$ as in Isaacs [7]. By choice of $G, \zeta$ is faithful and so $K^{\perp}=Z(K)$ and $Z(K)=Z(G)$ by choice of $K$. Thus 《U, $\rangle$ is nondegenerate on $K / Z$. This implies, [7], that $(K, Z, \tau)$ is a fully ramified triple and it follows that $G=\mathscr{J}_{G}(\tau)$. But now $(G, K, \tau)$ is fully ramified by Gagola [4, 2.2a] and Isaacs [7, 8.2] implies a central extension of $G / K$ is of central type and has the same nonabelian composition factors as $G$, contrary to the choice of $G$.

Lemma 2.3. $C_{G}(K)=C_{G}(K / Z)$.

Proof. Let $A=C_{G}(K / Z) / C_{G}(K)$. Then $A$ stabilizes the normal series $K>Z>1$ and so is a $p$-group. Let $\left\{a_{1} \cdots a_{s}\right\}$ be a minimal set of generators for $A$. Consider the commutator map $\left[a_{i},-\right]$ : $K \rightarrow Z$. Since $Z$ is cyclic ( $\zeta$ is faithful by choice of $G$ ), $K / C_{K}\left(a_{i}\right)$ is cyclic also. But $C_{K}\left(a_{i}\right) \geqq Z$ and $K / Z$ is elementary abelian, so $K / C_{K}\left(a_{i}\right)$ order 1 or $p$. It follows that $\left[K: C_{K}(A)\right]=\left[K: \cap C_{K}\left(a_{i}\right)\right] \leqq p^{s}$, and by Burnside's basis theorem [5, III. 3.2], $\left[K: C_{K}(A)\right] \leqq p^{s} \leqq[A: \Phi(A)] \leqq$ $|A|$.

Since $C_{K}(A)$ is $G$-invariant $C_{K}(A)=K$ or $Z$. If $C_{K}(A)=K$ the lemma holds, so suppose $C_{K}(A)=Z$. Let $K^{*}$ be the dual group of $K$ and let $V \leqq K^{*}$ be the set of characters vanishing on $Z$. If a power $\tau^{l}=\tau \otimes \cdots \otimes \tau$ of $\tau$ is in $V$, then $\zeta^{l}=\left(\left.\tau\right|_{z}\right)^{l}=1$ and $|Z| \mid l$, as $\zeta$ is faithful. Thus $K^{*}=\langle\tau, V\rangle$ and $C_{G}(V) \cap \mathscr{J}_{G}(\tau)=C_{G}\left(K^{*}\right)$. However, $C_{G}(K)=C_{G}\left(K^{*}\right)$ and $C_{G}(V)=C_{G}(K / Z)$, so $C_{G}(K / Z) \cap \mathscr{J}_{G}(\tau)=$ $C_{G}(K)$. Now $|A| \geqq\left[K: C_{K}(A)\right]=[K: Z]$ implies $G=\mathscr{J}_{G}(\tau) C_{G}(K / Z)$. Since $A_{G}=C_{G}(K / Z) /\left(C_{G}(K / Z) \cap \mathscr{I}_{G}(\tau)\right)$ is a $p$-group, the nonabelian composition factors of $G$ are also composition factors of $\mathscr{I}_{G}(\tau)$. Once again $\left(\mathscr{F}_{G}(\tau), K, \tau\right)$ is a fully ramified triple [4, 2.2a] and [7, 8.2] implies a central extension of $\mathscr{J}_{G}(\tau) / K$ is of central type. Since $G=\mathscr{J}_{G}(\tau) C_{G}(K / Z), \mathscr{J}_{G}(\tau)$ has the same nonabelian composition factors as $G$, contrary to the choice of $G$.

Lemma 2.4. Let $K^{*}$ denote the dual group of $K$ and $V \leqq K^{*}$ denote the characters vanishing on $Z$. If $H$ is a nonsolvable subnormal subgroup of $G$ containing $C_{G}(K)$ then $\bar{H}=H / C_{G}(K)$ violates hypothesis (1.2) and $H^{1}(\bar{H}, V) \neq 0$.

Proof. By Lemma 2.3 $\bar{H}$ acts faithfully on $V$. The constituents of the induced character $\zeta^{K}$ are $\tau V$, by $[8,6.17]$. Therefore $\tau^{h-1}=$ $\tau^{h} \tau^{-1} \in V$ for each $h \in \bar{H}$.

Consider the split extension $\bar{H} \ltimes V$ (with multiplication 
$\left.\left(h_{1} v_{1}\right)\left(h_{2} v_{2}\right)=\left(h_{1} h_{2}, v_{1}^{h_{2}} v_{2}\right)\right)$. Then $\phi: \bar{H} \rightarrow \bar{H} \ltimes V$ defined by $\phi(h)=\left(h, \tau^{h-1}\right)$ is an isomorphism. If $\phi(\bar{H})$ is conjugate to $\bar{H}=\{(h, 1) \mid h \in \bar{H}\}$, say $\phi(\bar{H})^{(g, x)}=\bar{H}$, then $\tau x^{-g^{-1}} \in \tau V$ is $H$-invariant. Thus $H \leqq \mathscr{F}_{G}\left(\tau x^{-g^{-1}}\right)$, and all composition factors of $H$ are composition factors of $G$. Again $\left(\mathscr{J}_{G}\left(\tau x^{-g^{-1}}\right), K, \tau x^{g^{-1}}\right)$ is fully ramified, contrary to the choice of $G$.

This shows $\bar{H} \ltimes V$ contains at least two conjugacy classes of complements to $V$ and so $H^{1}(\bar{H}, V) \neq 0$.

By Clifford's theorem $[8,17.3] G$ acts transitively on $\tau V$ and this set has $p$-power order. It follows [5, II. 1.5] that every $H$ orbit on $\tau V$ has $p$-power order. Suppose $I \leqq H$ is the stabilizer of $\sigma \in \tau V$. Then $I=\mathscr{J}_{H}(\sigma) \neq H$ by choice of $G$. However, $I=H \cap \mathscr{J}_{G}(\sigma)$ is subnormal in $\mathscr{I}_{G}(\sigma)$ and the choice of $G$ forces the nonabelian composition factors of $I$ to fail (1.1). This shows $\bar{H}$ fails hypothesis (1.2).

LEMma 2.5. Let $T$ be the maximal solvable normal subgroup of G. Then $T=C_{G}(K)$.

Proof. Since $C_{G}(K) \triangleleft G$ and $C_{G}(K) \leqq \mathscr{F}_{G}(\tau)$, the minimality of $G$ implies $C_{G}(K)$ is solvable and so $T \geqq C_{G}(K)$.

Suppose $T \neq C_{G}(K)$ and let $R$ be a minimal normal subgroup of $G$ among those containing $C_{G}(K)$ and contained in $T$. Then $R$ acts completely reducibly on $V$ (as in Lemma 2.4) by Clifford's theorem [8, 17.3]. By Lemma 2.3 $C_{V}(R)=1$ and $R / C_{G}(K)$ is a $p^{\prime}$-subgroup. It follows that $N_{V \cdot \bar{G}}(R)=\bar{G}$ where $\bar{G}=G / C_{G}(K)$. Now the SchurZassenhaus theorem implies $H^{1}(R, V)=0$ and so $0=H^{1}\left(N_{V \in \bar{G}}(\bar{R}), V\right)=$ $H^{1}(G, V)$ contrary to Lemma 2.4 .

THEOREM 2.6. Let $G$ be a nonsolvable group of central type of minimal order among those possessing only composition factors that satisfy (1.1). Let $Z=Z(G)$ and let $K$ be a minimal subgroup of $G$ among those properly containing $Z$. Then $F\left(G / C_{G}(K)\right)=1$ and no component of $G / C_{G}(K)$ satisfies (1.2) for the prime $p$ dividing $K / Z$.

Proof. By Theorem 2.1 and Lemma 2.2, $K \leqq C_{G}(K)$. By Lemma 2.3 $\bar{G}=G / C_{G}(K)$ acts faithfully on $V$ where $V$ is the subgroup of the dual group of $K$ consisting of characters with kernel containing $Z$ and the fitting group $F(\bar{G})$ is trivial by Lemma 2.5.

If $S$ is a component of $\bar{G}$, then there is a subnormal subgroup $H \geqq C_{G}(K)$ such that $\bar{H} \cong S$, and Lemma 2.4 applies.

Theorem 2.7. Let $G$ and $K$ be as in 2.6. Then PSL $(2, q)$ is not a component of $G / C_{G}(K)$. 
Proof. Suppose $H \geqq C_{G}(K)$ and $H / C_{G}(K) \cong \operatorname{PSL}(2, q)$ is a component of $\bar{G}=G / C_{G}(K)$. Observe that $H$ satisfies the hypotheses of Lemma 2.4 and recall the proof of this lemma. It was shown that each $H$-orbit on $\tau V$ has nontrivial $p$-power order.

However, the subgroups of PSL $(2, q)$ are all known [5, II. 8.27] and only for certain $q$ (see 3.2) does there exist a proper subgroup of $p$-power index and in each of these cases (except PSL $(2,7)$, $p=7$ ), the subgroup is unique up to conjugacy.

Assume at least one of $p$ and $q$ is not 7 and take $H \geqq I \geqq C_{G}(K)$ so $[H: I]$ is a nontrivial power of $p$. Then $I$ fixes an element of each $H$-orbit on $\tau V$. Therefore $|\tau V|=\left|C_{\tau V}(I)\right|[H: I]$ and so $\left[K^{*}: C_{K^{*}}(I)\right] \leqq[H: I]$. Now $H=\left\langle I, I^{h}\right\rangle$ for $h$ in $H$ but not in $I$, so

$$
\left[K^{*}: C_{K^{*}}(H)\right]=\left[K^{*}: C_{K^{*}}(I) \cap C_{K^{*}}\left(I^{h}\right)\right] \leqq[H: I]^{2} \text {. }
$$

We have shown $\bar{H}=H / C_{G}(K)$ acts faithfully on $W=K^{*} / C_{K^{*}}(H)$ and $|W| \leqq[H: I]^{2}$.

In case $[H: I]=2^{m}, q$ is a Mersene prime and $I$ is the normalizer of a Sylow $q$-group $Q$. Consequently, an element $A$ of order $(q-1) / 2$ normalizing $Q$ acts faithfully on $[W, Q]$. Since $Q$ acts on $[W, Q]$ as the full multiplicative group of $G F\left(2^{m}\right)$, this implies $(q-1) / 2 \mid m$, and so $m=3$. Since $A$ normalizes a second Sylow $q$-subgroup $Q_{1}$ and centralizes $C_{W}\left(Q_{1}\right)$, it follows that $|W|=16$ and $W$ has an irreducible submodule of order 8. This situation cannot occur in $G$ since $S$ is subnormal in $G$ and hence acts completely reducibly on $V$ by Clifford's theorem.

In case $[H: I]=9, q=8$ and we have a homeomorphism of $\operatorname{PSL}(2,8)$ into SL $(4,3)$ contrary to the fact that 7 does not divide $|\operatorname{SL}(4,3)|$.

In case $[H: I]=p, q$ is a power of 2 and we have $\mathrm{SL}(2, q)$ as a subgroup of $\mathrm{SL}(2, p)$. This is impossible. (Even in case $p=5$, $q=4$, SL $(2,5)$ has no subgroup of order 60 .)

We are left with the case $p=q=7$. Here there are exactly two possible conjugacy classes for $I$ and so we may choose $I$ so $|\tau V| \leqq 2\left|C_{\tau V}(I)\right|[H: I]$ and so $\left[K^{*}: C_{K^{*}}(I)\right] \leqq 2[H: I]$. However, both $[H: I]$ and $\left[K^{*}: C_{K^{*}}(I)\right]$ are powers of 7 , so we have $\left[K^{*}: C_{K^{*}}(I)\right]$ just as above. It follows that PSL $(2,7)$ is a subgroup of $\operatorname{SL}(2,7)$, contrary to the fact that SL $(2,7)$ is perfect.

\section{Hypothesis (1.1) and (1.2) and the known simple groups.}

TheORem 3.1. If $S$ is a simple alternating group, group of Lie type or one of the first 26 sporadic simple groups, $S$ satisfies hypothesis (1.1).

Proof. In case $S$ is an alternating group, this follows from 
Bertram's postulate $[11,8.6]$ and the fact that $\mid$ Out $S|| 4$.

Suppose $S$ is of Lie type having characteristic $p$. The $p^{\prime}$ part of $|S|$ is a product of terms of the form $\left(p^{i}-1\right) / k_{i}$ where $k_{i}=1$ or $p^{j}-1$ for some $j<i$. Let $m$ be the maximal value of $i$ for which there is such a factor.

Assume there is a $p$-primitive prime divisor $r$ of $\left(p^{m}-1\right)$. Then a Sylow $r$-subgroup of $S$ is cyclic and Out $S$ is generated by diagonal, graph and field automorphisms, Steinberg [13]. The group of diagonal automorphisms $D$ has order dividing the order of the multiplicative group of the underlying field and so $r \nmid|D|$. Fermat's theorem and $p^{m} \equiv 1(\bmod r)$ imply $(r-1) \mid m$ and so $r$ does not divide the order of the group of field automorphisms. Hypothesis (1.1) now follows unless perhaps $r=3$ and the diagram of $S$ has 3 -fold symmetry, i,e., if $S=D_{4}\left(p^{t}\right)$. Thus we reduce to the case $S=D_{4}\left(p^{t}\right), m=4 t$ and 3 is the only $p^{t}$-primitive prime divisor of $p^{m}-1$. Then $p^{2 t}+1$ has the form $2^{a} \cdot 3^{b}$ and consideration of the squares modulo 12 leads to a contradiction. This shows a group of Lie type satisfies hypothesis (1.1) unless perhaps $p^{m}-1=63$ or $p$ is a Mersene prime and $m=2$, [15].

In the first case, inspection of the group order formulae leads to the possibilities: $\operatorname{PSL}(2,8), \operatorname{PSL}(3,4), \operatorname{PSL}(6,2), \operatorname{PSP}(6,2)$, $P \Omega(5,2), P \Omega^{+}(8,2)$ and the solvable group $\operatorname{PSU}(3,2)$. For these groups, the primes $r=7,5,31,5,5$ and 7 respectively satisfy hypothesis (1.1). In the second case $S=\operatorname{PSL}(2, p)$ is the only possibility and it has a cyclic Sylow $p$-subgroup.

Suppose finally that $S$ is one of the first 26 sporadic groups. Then inspection of the list of orders of $S$, Rudvalis and Hurley [6] reveals that $|S|$ is divisible by a prime $r>7$ to the first power, and inspection of the list of $\mid$ Out $S \mid$, Aschbacher and Seitz [1, Table 1] shows $\mid$ Out $S \| 2$.

THEOREM 3.2. Let $S$ be as in 3.1. If $S$ fails hypothesis 1.2 then $S=\mathrm{PSL}\left(2,2^{m}-1\right)$ where $2^{m}-1$ is prime or PSL $\left(2,2^{m}\right)$ where $2^{m}+1$ is prime or 9 .

Proof. Suppose $S$ is an alternating group. The group $A_{5} \cong$ PSL $(2,4)$ is exceptional. Observe that $A_{6}$ possesses no subgroups of prime power index. Fix a prime $p$ and choose $n>6$ minimal so that $A_{n}$ has a subgroup $I$ of $p$-power index. If $I$ acts transitively $A_{n} / A_{n-1}$ then $A_{n-1} \cap I$ is of $p$-power index in $A_{n-1}$, contrary to choice of $n$. Therefore, $I$ has at least two orbits on $A_{n} / A_{n-1}$ and so $|I| \mid k !(n-k) ! / 2$ for some $1 \leqq k \leqq n$. (No element of $I$ can induce an even permutation on one $I$-orbit and an odd permutation on the complement of this orbit.) Therefore, the binomial coefficient $\left(\begin{array}{l}n \\ k\end{array}\right)$ 
divides $\left[A_{n}: I\right]$ and is a $p$-power. This forces $k=1$ and $n$ itself to be a prime power. This shows the only subgroups of $p$-power index in $A_{n}$ are $A_{n-1}$ in case $n$ is a $p$-power and so $A_{n}$ satisfies hypothesis (1.2) for $n>6$ since $A_{n-1}$ is a simple group satisfying hypothesis (1.1) by Theorem 3.1.

Next suppose $S$ is a group of Lie type and characteristic $r$. Suppose $I \leqq S$ is of $p$-power index. In case $r=p$, Sylow $p$-subgroup of $G$ acts transitively on $G / K$ and so $K$ acts transitively on the set of Sylow $p$-subgroups of $S$. Thus Theorem A of Seitz [12] applies. None of the possible groups $I$ in his list has a composition factor violating (1.1) and the only cases where $I$ is solvable appear in our list. Next assume $r \neq p$. Then a lemma of Tits $[12,1.6]$ implies that a maximal subgroup $K$ containing $I$ is parabolic. Just as in the proof of 3.1, let $m$ be the maximum value of $i$ for which $|S|$ has a factor of the form $\left(r^{i}-1\right) /\left(r^{i}-1\right), i>j$.

In case $\left(r^{m}-1\right)$ has an $r$-primitive prime divisor $s$ then $s$ divides the index of every parabolic subgroup of $S$ and so $r=s$ and $K=I$ corresponds to an extremal node in the associated diagram. Now the nonsolvable composition factors of $I$ are groups of Lie type and so they satisfy hypothesis (1.1). The only way $I$ can be solvable is if $S$ has $(B, N)$ rank $\leqq 2$ and the possibilities appear in our list.

In case $p^{m}-1$ has no $r$-primitive prime divisors then either $S=\operatorname{PSL}\left(2,2^{m}-1\right)$ (which appears in our list) or $p^{m}-1=63$, Zsigmondy [15], and $S$ is one of seven explicit groups. Of these only PSL $(2,8)$ has a subgroup of prime power index.

A great deal is known about the 26 known sporadic groups and none of them has a solvable subgroup of prime power index, see Aschbacher and Seitz [1].

\section{REFERENCES}

1. M. Aschbacher and G. Seitz, On groups of standard component type, Osaka J. Math., 13 (1976), 439-482.

2. F. DeMeyer and G. J. Janusz, Finite groups with an irreducible representation of large degree, Math. Z., 108 (1969), 1-153.

3. T. M. Gagen, Topics in Finite Groups, London Math. Soc. Lecture notes, 16 (1976), Cambridge Univ. Press, Cambridge, England.

4. S. M. Gagola, Characters fully ramified over a normal subgroup, Pacific J. Math., 55 (1974), 107-126.

5. B. Huppert, Endliche Gruppen I, Springer, New York, 1967.

6. J. Hurley and A. Rudvalis, Finite simple groups, Amer. Math. Monthly, 84 (1977), 693-713.

7. I. M. Isaacs, Characters of solvable and symplectic groups, Amer. J. Math., 95 (1973), 594-635.

8. - Character Theory of Finite Groups, Academic Press, New York, 1976.

9. N. Ito, On the degrees of irreducible representations of a finite group, Nagoya Math. J., 3 (1951), 5-6. 
10. N. Iwohori and H. Matsumoto, Several remarks on projective representations of finite groups, J. Fac. Sci. U. Tokyo, Sect. I, 10 (1964), 129-146.

11. I. Niven and H. Zuckerman, An Introduction to the Theory of Numbers, Wiley and Sons, New York, 1960.

12. G. M. Seitz, Flag-transitive subgroups of Chevellary groups, Annal of Math., 97 (1973), 26-57.

13. R. Steinberg, Automorphisms of finite linear groups, Canad. J. Math., 12 (1960), 606-615.

14. J. E. Yellen, On the solvability of groups of central type, PAMS, 52 (1975), 50-54.

15. K. Zsigmondy, Zuer Theorie der Potenzreste, Monatsch. Math. u. Phys., 3 (1892), 265-284.

Received June 2, 1978 and in revised form November 13, 1978. Research of the first author was partially supported by NSE Grant MCF 7701976.

Colorado State University

FORT COLLINS, CO 80523

NAD

SUNY

FREDONIA, NY 14063 


\section{PACIFIC JOURNAL OF MATHEMATICS}

\section{EDITORS}

Donald BABBITT (Managing Editor)

University of California

Los Angeles, CA 90024

HUGo RossI

University of Utah

Salt Lake City, UT 84112

C. C. MOORE and ANDREW OGG

University of California

Berkeley, CA 94720

\section{J. DuGUNDJI}

Department of Mathematics

University of Southern California

Los Angeles, CA 90007

R. FINN and J. Milgram

Stanford University

Stanford, CA 94305

ASSOCIATE EDITORS
E. F. BECKENBACH
B. H. NeumanN
F. WOLF
K. YoSHIDA

\section{SUPPORTING INSTITUTIONS}

\author{
UNIVERSITY OF BRITISH COLUMBIA \\ CALIFORNIA INSTITUTE OF TECHNOLOGY \\ UNIVERSITY OF CALIFORNIA \\ MONTANA STATE UNIVERSITY \\ UNIVERSITY OF NEVADA, RENO \\ NEW MEXICO STATE UNIVERSITY \\ OREGON STATE UNIVERSITY \\ UNIVERSITY OF OREGON
}

\author{
UNIVERSITY OF SOUTHERN CALIFORNIA \\ STANFORD UNIVERSITY \\ UNIVERSITY OF HAWAII \\ UNIVERSITY OF TOKYO \\ UNIVERSITY OF UTAH \\ WASHINGTON STATE UNIVERSITY \\ UNIVERSITY OF WASHINGTON
}

The Supporting Institutions listed above contribute to the cost of publication of this Journal, but they are not owners or publishers and have no responsibility for its content or policies.

Mathematical papers intended for publication in the Pacific Journal of Mathematics should be in typed form or offset-reproduced, (not dittoed), double spaced with large margins. Please do not use built up fractions in the text of the manuscript. However, you may use them in the displayed equations. Underline Greek letters in red, German in green, and script in blue. The first paragraph or two must be capable of being used separately as a synopsis of the entire paper. Please propose a heading for the odd numbered pages of less than 35 characters. Manuscripts, in triplicate, may be sent to any one of the editors. Please classify according to the scheme of Math. Reviews, Index to Vol. 39. Supply name and address of author to whom proofs should be sent. All other communications should be addressed to the managing editor, or Elaine Barth, University of California, Los Angeles, California, 90024.

50 reprints to each author are provided free for each article, only if page charges have been substantially paid. Additional copies may be obtained at cost in multiples of 50 .

The Pacific Journal of Mathematics is issued monthly as of January 1966. Regular subscription rate: $\$ 84.00$ a year (6 Vols., 12 issues). Special rate: $\$ 42.00$ a year to individual members of supporting institutions.

Subscriptions, orders for numbers issued in the last three calendar years, and changes of address should be sent to Pacific Journal of Mathematics, P.O. Box 969, Carmel Valley, CA 93924, U.S.A. Older back numbers obtainable from Kraus Periodicals Co., Route 100, Millwood, NY 10546.

PUBLISHED BY PACIFIC JOURNAL OF MATHEMATICS, A NON-PROFIT CORPORATION

Printed at Kokusai Bunken Insatsusha (International Academic Printing Co., Ltd.). 8-8, 3-chome, Takadanobaba, Shinjuku-ku, Tokyo 160, Japan.

Copyright (C) 1979 by Pacific Journal of Mathematics Manufactured and first issued in Japan 


\section{Pacific Journal of Mathematics}

\section{Vol. 82 , No. 2 \\ February, 1979}

Krishnaswami Alladi and Paul Erdős, On the asymptotic behavior of large prime

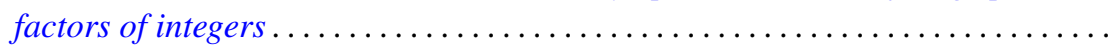

Alfred David Andrew, A remark on generalized Haar systems in $L_{p}$,

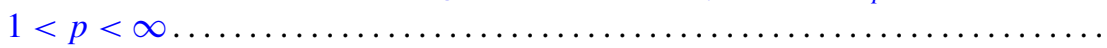

John M. Baker, A note on compact operators which attain their norm . . ........

Jonathan Borwein, Weak local supportability and applications to

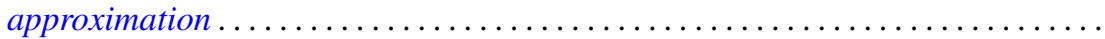

Tae Ho Choe and Young Soo Park, Wallman's type order compactification ........

Susanne Dierolf and Ulrich Schwanengel, Examples of locally compact

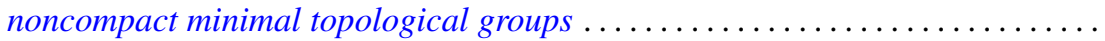

Michael Freedman, A converse to (Milnor-Kervaire theorem) $\times R$ etc. . . . . . . .

George Golightly, Graph-dense linear transformations ..................

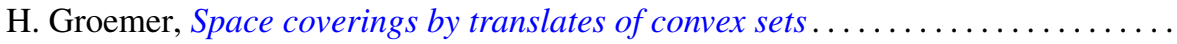

Rolf Wim Henrichs, Weak Frobenius reciprocity and compactness conditions in

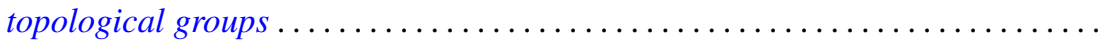

Horst Herrlich and George Edison Strecker, Semi-universal maps and universal

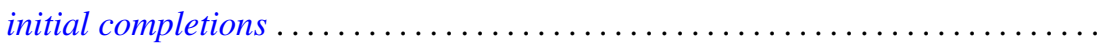

Sigmund Nyrop Hudson, On the topology and geometry of arcwise connected,

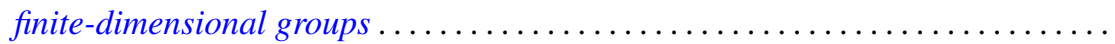

K. John and Václav E. Zizler, On extension of rotund norms. II .............

Russell Allan Johnson, Existence of a strong lifting commuting group of transformations. II.

Bjarni Jónsson and Ivan Rival, Lattice varieties covering the smallest nonmodular variety

Grigori Abramovich Kolesnik, On the order of Dirichlet L-functions .

Robert Allen Liebler and Jay Edward Yellen, In search of nonsolvable groups of

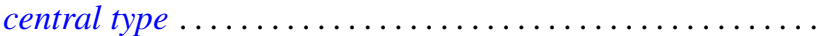

Wilfrido Martínez T. and Adalberto Garcia-Maynez Cervantes, Unicoherent plane Peano sets are $\sigma$-unicoherent ...

M. A. McKiernan, General Pexider equations. I. Existence of injective

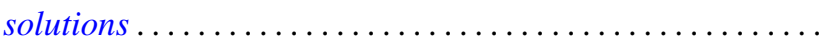

M. A. McKiernan, General Pexider equations. II. An application of the theory of webs.

Jan K. Pachl, Measures as functionals on uniformly continuous functions . .

Lee Albert Rubel, Convolution cut-down in some radical convolution algebras ...

Peter John Slater and William Yslas Vélez, Permutations of the positive integers

with restrictions on the sequence of differences. II . . .

Raymond Earl Smithson, A common fixed point theorem for nested spaces ....

Indulata Sukla, Generalization of a theorem of McFadden .... . . .

Jun-ichi Tanaka, A certain class of total variation measures of analytic measures.

Kalathoor Varadarajan, Modules with supplements .............. 